\title{
A Closed Loop Stability Analysis and Parameter Selection of the Particle Swarm Optimization Dynamics for Faster Convergence
}

\author{
Nayan R. Samal, Amit Konar, Swagatam Das and Ajith Abraham
}

\begin{abstract}
This paper presents an alternative formulation of the PSO dynamics by a closed loop control system, and analyzes the stability behavior of the system by using Jury's test and root locus technique. Previous stability analysis of the PSO dynamics was restricted because of no explicit modeling of the non-linear element in the feedback path. In the present analysis, the nonlinear element model of the non-linear element is considered for closed loop stability analysis. Unlike the previous works on stability analysis, where the acceleration coefficients have been combined into a single term, this paper considered their separate existence for determining their suitable range to ensure stability of the dynamics. The range of parameters of the PSO dynamics, obtained by Jury's test and root locus technique were also confirmed by computer simulation of the PSO algorithm.
\end{abstract}

\section{INTRODUCTION}

$\mathrm{P}$ ioneered by Eberhart and Kennedy, the Particle Swarm Optimization (PSO) algorithm [1] is a population based intelligent search, where individuals called particles are candidate solutions to a given optimization problem. In a PSO algorithm, particles adapt their velocities and positions in a multidimensional search space until one or more particles converge to the global optima in the search space. Empirical studies [2] on PSO reveals that its parameters well known as 'inertia factor' and 'acceleration coefficients' are all positive constants. There also exist some mathematical studies to guideline parameter selection in PSO dynamics [3]. Currently, the PSO dynamics is modeled by a feedback control system [4], where the forward path represents the dynamics of an individual particle and the feedback path includes a non-linear element. Unfortunately existing stability analysis of PSO dynamics is restricted, because of the absence of a suitable representation of the non-linear element in the feedback path. In this paper, we consider possible representation of the non-linear element and perform the stability analysis of the closed loop system using Jury's test [5] and root-locus technique [6].

Early parameter selection studies [3], [7], [8] of PSO dynamics was undertaken through computer simulations. The first mathematical analysis on stability for the PSO algorithm

Nayan R. Samal is with the ETCE Department, Jadavpur University, Kolkata 700032, India; e-mail: nayan@ softcomputing.net

Amit Konar is with the ETCE Department, Jadavpur University, Kolkata 700032, India; e-mail: amit@softcomputing.net

Swagatam Das is with the ETCE Department, Jadavpur University, Kolkata 700032, India; e-mail: swagatam@softcomputing.net

Ajith Abraham is with the Center of Excellence for Quantifiable Quality of Service (Q2S), Norwegian University of Science and Technology, Norway; email: ajith.abraham@ieee.org was undertaken in [3]. Clerc and Kennedy represented the PSO dynamics as a second order linear system and determined the condition for stability using eigenvalue analysis of the state transition matrix [3]. Van den Bergh [7] also performed a stability analysis for the PSO dynamics to identify regions in the parametric space to guarantee stability of the system. There exist a few other relevant works on parameter selection of the PSO dynamics [9], [10], [11].

In this paper, we propose a closed loop stability analysis for the PSO dynamics. The analysis differs from the existing works [4], [9] by the following counts. Unlike the previous works, we here explicitly represent the non-linear element by mathematical models and perform stability analysis for the closed loop system using the model. Because of a specialized state space representation of our model, here the error detector continuously attempts to minimize the difference between the global best position of all the particles and the local best position by the particle under considerations. Further, the deviation of the current position of the particle from the global best position here is considered as the response of the PSO dynamics. The non-linear element here has been modeled to provide the current local best position from its last value and the current position of the particle.

Jury's stability test criteria have been applied on the characteristic equation of the closed loop system, to determine the conditions for stability for the closed loop dynamics. These conditions envisage the suitable parametric space for the PSO dynamics. The root locus analysis for the closed loop PSO system undertaken here provides suitable range for the acceleration coefficients that satisfy the stability conditions for two possible forms of representations of the non-linear element. Faster convergence of the PSO dynamics is attained here by determining the locations of the dominant closed loop poles [6] in the root locus. These dominant poles dictate the best parameter settings for the PSO dynamics for faster convergence. The results of root locus analysis are validated by test runs of the PSO algorithm on standard 21 benchmark functions [12].

The paper is organized as follows. The basic PSO dynamics and its state space analysis is illustrated in Section II. The closed loop representation of the PSO dynamics and in particular modeling of the non-linear element is considered in Section III. Stability analysis by Jury's test is performed in Section IV. Parameter selection for the PSO dynamics by root locus technique is undertaken in section V. Computer simulations supporting the established results of Section V are given in Section VI. Conclusions are listed in Section VII. 


\section{ThE PSO DyNAMICS AND ITS STATE SPACE REPRESENTATION}

The PSO algorithm involves the dynamics of a group of particles over a d-dimensional fitness landscape. Each particle embodies a trial solution of the objective function to be optimized. The motion of a particle may be conveniently represented in one dimensional space as follows:

$$
\begin{gathered}
v_{t}=\omega \cdot v_{t-1}+\alpha_{t-1}^{l} \cdot\left(p^{l}-x_{t-1}\right)+\alpha_{t-1}^{g} \cdot\left(p^{g}-x_{t-1}\right) \\
x_{t}=x_{t-1}+v_{t}
\end{gathered}
$$

where $v_{t}$ is the velocity of the particle at $t^{\text {th }}$ iteration, $x_{t}$ is the particle position at the $t^{\text {th }}$ iteration, $p^{l}$ is the personal (local) best position of the particle so far achieved, $p^{g}$ is the global best position among all particles. The parameters in the PSO dynamics that we need to determine for stability analysis include the inertia factor $\omega$, the acceleration coefficients for the local best $\alpha_{t}^{l}$ and that for the global best position $\alpha_{t}^{g}$. Following the classical analysis and empirical simulation results, we know $\omega, \alpha^{l}$ and $\alpha^{g}$ are $>0$ and $\alpha^{l}$ and $\alpha^{g}$ are positive and bounded random numbers.

$$
\text { Let } \overline{x_{t}}=p^{g}-x_{t}
$$

Hence, $p^{l}-x_{t}=p^{l}+\left(\overline{x_{t}}-p^{g}\right)$

$$
=\left(p^{l}-p^{g}\right)+\overline{x_{t}}
$$

From equations (1) and (3) we obtain:

$$
\begin{aligned}
& v_{t}=\omega \cdot v_{t-1}+\alpha_{t-1}^{l}\left[\left(p^{l}-p^{g}\right)+\bar{x}_{t-1}\right] \\
& \quad+\alpha_{t-1}^{g} \cdot \bar{x}_{t-1} \\
& =\omega \cdot v_{t-1}+\left(\alpha_{t-1}^{l}+\alpha_{t-1}^{g}\right) \bar{x}_{t-1}+\alpha_{t-1}^{l}\left(p^{l}-p^{g}\right) \\
& =\omega \cdot v_{t-1}+\alpha_{t-1} \cdot \bar{x}_{t-1}+\alpha_{t-1}^{l}\left(p^{l}-p^{g}\right)
\end{aligned}
$$

where $\alpha_{t-1}=\alpha_{t-1}^{l}+\alpha_{t-1}^{g}$.

Further, $\overline{x_{t}}=p^{g}-x_{t}$

$$
\begin{gathered}
=p^{g}-\left(x_{t-1}+v_{t}\right) \\
=p^{g}-x_{t-1}-v_{t} \\
=p^{g}-x_{t-1}-\omega \cdot v_{t-1}-\alpha_{t-1} \cdot \bar{x}_{t-1}-\alpha_{t-1}^{l}\left(p^{l}-p^{g}\right)
\end{gathered}
$$

[by substitution of (4)]

$$
=\left(1-\alpha_{t-1}\right) \cdot \bar{x}_{t}-\omega \cdot v_{t-1}-\alpha_{t-1}^{l}\left(p^{l}-p^{g}\right)
$$

Representing equations (4) and (7) in vector matrix form we obtain:

$$
\begin{aligned}
& \left(\begin{array}{l}
\bar{x}_{t} \\
v_{t}
\end{array}\right)=\left(\begin{array}{cc}
\left(1-\alpha_{t-1}\right) & -\omega \\
\alpha_{t-1} & \omega
\end{array}\right)\left(\begin{array}{l}
\bar{x}_{t-1} \\
v_{t-1}
\end{array}\right)+ \\
& \left(\begin{array}{c}
\alpha_{t-1}^{l} \\
-\alpha_{t-1}^{l}
\end{array}\right) \cdot\left(p^{g}-p^{l}\right)
\end{aligned}
$$

This is the state equation of the basic PSO algorithms. Here,

$$
A=\left(\begin{array}{cc}
\left(1-\alpha_{t-1}\right) & -\omega \\
\alpha_{t-1} & \omega
\end{array}\right) \text { is the system matrix. }
$$

The stability of the dynamics can be envisaged from the Eigen values $(\lambda)$ of the above matrix. Setting det $(A-\lambda I)=0$, we obtain

$$
\lambda=\frac{\left(1-\alpha_{t-1}+\omega\right) \pm \sqrt{\left(1-\alpha_{t-1}-\omega\right)^{2}-4 \omega}}{2}
$$

Defining $\alpha_{t-1}-\omega-1=\delta$,

$$
\lambda=\frac{-\delta \pm \sqrt{\delta^{2}-4 \omega}}{2}
$$

\section{III.CLOSED LOOP REPRESENTATION OF THE PSO DYNAMICS}

Stability of the dynamics now can be ascertained from the Eigen values of the above system. The state equation (8) can be described in terms of feedback control system model as outlined in Figure 1.

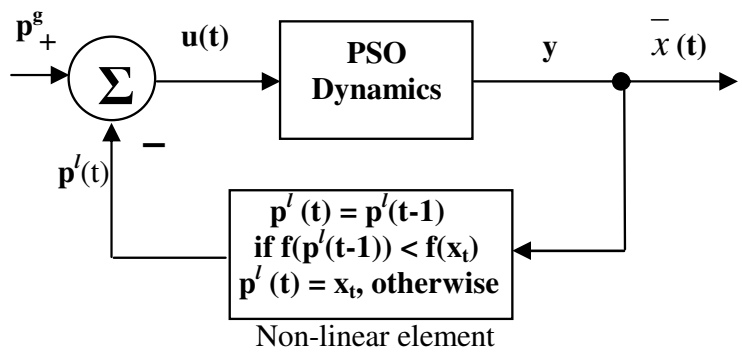

Fig.1. The feedback control representation of particle dynamics

Here, the non-linear element determines $p_{t}^{l}$ from its previous value $p_{t-1}^{l}$ and $x_{t}$. The non-linear function is formally represented by

$$
\begin{aligned}
p_{t}^{l} & =p_{t-1}^{l} \text { if } f\left(p_{t-1}^{l}\right)<f\left(x_{t}\right) \\
& =x_{t}, \text { otherwise }
\end{aligned}
$$

where $f($.$) denotes the fitness function of the rough non-$ linear search space. The PSO dynamics being linear, can be represented in the form of a transfer function.

Let $M(z)=\frac{Y(z)}{U(z)}$ 
Since the state equation consists of two variables $\bar{x}_{t}$ and $v_{t}$ and we are interested to feedback $\bar{x}_{t}$ only, we select a matrix $C=\left(\begin{array}{ll}1 & 0\end{array}\right)$.

For the output equation $\mathrm{Y}=\mathrm{C}\left(\begin{array}{ll}\bar{x}_{t} & v_{t}\end{array}\right)^{T}$

$M(z)$ can be evaluated by;

$$
\begin{aligned}
& C(z I-A)^{-1} B \\
& \quad=\left(\begin{array}{ll}
1 & 0
\end{array}\right)\left(\begin{array}{cc}
z-1+\alpha_{t-1} & \omega \\
-\alpha_{t-1} & z-\omega
\end{array}\right)^{-1}\left(\begin{array}{c}
\alpha^{l}{ }_{t-1} \\
-\alpha^{l}{ }_{t-1}
\end{array}\right) \\
& \quad=\frac{1}{\Delta}\left(z \alpha^{l}{ }_{t-1}\right)
\end{aligned}
$$

Where $\Delta=(z-\omega)\left(z-1+\alpha_{t-1}\right)+\omega \alpha_{t-1}$

$$
=z^{2}+z\left(\alpha_{t}-w-1\right)+\omega
$$

Thus

$$
\frac{\bar{x}(z)}{u(z)}=\frac{z \alpha_{t-1}^{l}}{z^{2}+z\left(\alpha_{t-1}-\omega-1\right)+\omega}
$$

The non-linearity can be modeled by considering two possible values for $p_{t}^{l}$.

$$
p_{t}^{l} \leftarrow p_{t-1}^{l} \text { for } f\left(p_{t-1}^{l}\right)<f\left(x_{t}\right)
$$

and $\quad p_{t}^{l} \leftarrow x_{t}=\left(p^{g}-\bar{x}_{t}\right)$,otherwise.

The first condition can be modeled by using

$$
p_{t}^{l} \leftarrow \operatorname{Min}\left\{x_{t}\right\} \text {, for all } \mathrm{t}
$$

Since Min $\left\{x_{t}\right\}$ is not known, we presume $p_{t}^{l}$ as one of the previous $x_{t}$. Thus in Z-domain we write

$$
\begin{aligned}
p^{l}(z) & \leftarrow z^{-n} \bar{x}(Z), \mathrm{n} \geq 1 \\
& =z^{-n}\left(p^{g}-\bar{x}(z)\right)
\end{aligned}
$$

The second condition can be written as

$$
p^{l}(z) \leftarrow p^{g}-\bar{x}(Z)
$$

Based on equation (19) we modify the closed loop configuration of Figure 1, as given in Figure 2 (a). The equivalent representation of Figure 2 (a) is given in Figure 2 (b). The closed loop representation of equation (18) is given in Figure 3.

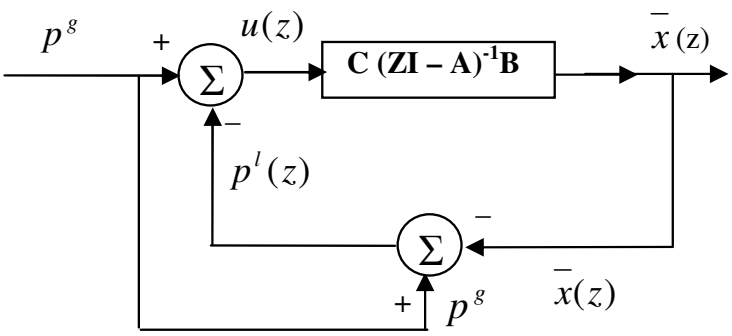

Fig.2 (a): Representation of the PSO closed loop dynamics by modeling non-linearity using equation (19)

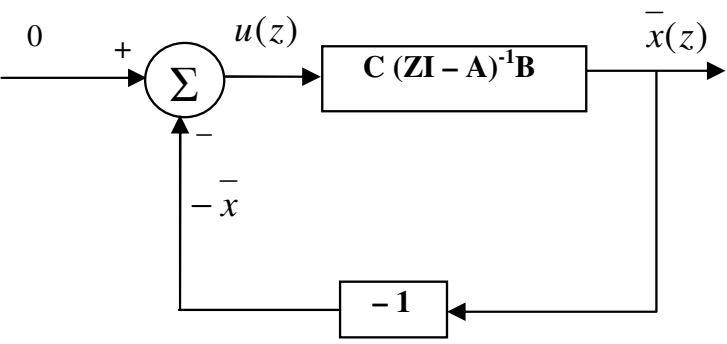

Fig.2 (b): An equivalent representation of Fig 2(a)

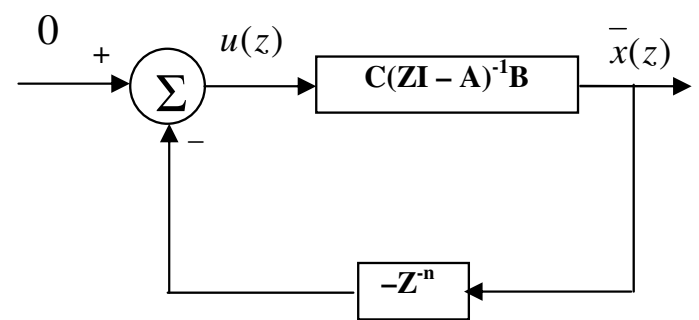

Fig.3: Similar representation of the PSO closed loop dynamics by modeling nonlinearity using equation (18)

Figure 3 was obtained from equation (18) by using the identity, $u(z)=p^{g}-\left(p^{g}-\bar{x}(z)\right) z^{-n}=z^{-n} \cdot \bar{x}(z)$, as $p^{g}$ is treated constant.

\section{IV.STABILITY ANALYSIS OF CLOSED LOOP SYSTEM}

The characteristic equation for the given system (Figure 2 (b)) is given by:

$$
1+C(Z I-A)^{-1} B .(-1)=0
$$

which yields

$$
\begin{aligned}
& F(Z)=1+z^{-1}\left(\alpha_{t-1}-\omega-1-\alpha_{t-1}^{l}\right)+z^{-2} w=0 \\
\Rightarrow & F(Z)=z^{2}+z\left(\alpha_{t-1}^{g}-\omega-1\right)+\omega
\end{aligned}
$$

According to Jury's test:

$$
\begin{aligned}
\mathrm{F}(1) & =1+\left(\alpha_{t-1}^{g}-\omega-1\right)+\omega>0 \\
& \Rightarrow \alpha_{t-1}^{g}>0 \\
\mathrm{~F}(-1) & =1-\left(\alpha_{t-1}^{g}-\omega-1\right)+\omega>0 \\
& \Rightarrow \alpha_{t-1}^{g}<2(1+\omega)
\end{aligned}
$$

Further by Jury's test $\left|a_{0}\right|<a_{2}$ which yields

$$
\Rightarrow \omega<1
$$

where $a_{i}$ is the co-efficient of $z^{i}$ in equation 19(b).

The range of $\alpha_{t-1}^{g}$ as obtained from (20) and (21) is,

$$
0<\alpha_{t-1}^{g}<2(1+\omega) \text { for all } \mathrm{t} .
$$

On the other hand, when we consider the closed loop dynamics of Figure 3, the characteristic equation is given by,

$$
\begin{aligned}
\mathrm{F}(\mathrm{Z}) & =1+\mathrm{C}(\mathrm{ZI}-\mathrm{A})^{-1} \mathrm{~B} \cdot \mathrm{Z}^{-\mathrm{n}}, \quad \mathrm{n} \geq 1 . \\
& =z^{n+2}+z\left(\alpha_{t-1}^{g}-\omega-1\right)+\omega=0
\end{aligned}
$$


Now by Jury's test, $\left|a_{0}\right|<a_{n+2}$ yields $\omega<1$.

$$
\begin{gathered}
\mathrm{F}(1)>0 \text { yields } 1+\left(\alpha_{t-1}^{g}-\omega-1\right)+\omega>0 \\
\Rightarrow \alpha_{t-1}^{g}>0 \\
\mathrm{~F}(-1)=-1-\left(\alpha_{t-1}^{g}-\omega-1\right)+\omega<0 \text {,for odd }(\mathrm{n}+2) \\
\Rightarrow \alpha_{t-1}^{g}>2 \omega \\
\text { Combining }(20) \text { and }(24) \\
2 \omega<\alpha_{t-1}^{g}<2(\omega+1)
\end{gathered}
$$

\section{V.RoOT LOCUS METHOD FOR STABILITY ANALYSIS FOR THE PSO DYNAMICS}

For convenience of the analysis, we first consider phase of the $G(Z) . H(Z)$ is $180^{\circ}$, where $G(Z)$ is the forward path gain and $\mathrm{H}(\mathrm{Z})$ is the feedback path gain. Setting $\mathrm{Z}=\mathrm{x}+\mathrm{j} \mathrm{y}$, we thus obtain

$$
\begin{gathered}
\angle G(z) \cdot H(z)= \\
\angle \frac{(x+j y) \alpha^{l}{ }_{t-1}}{(x+j y)^{2}+(x+j y)\left(\alpha_{t-1}-\omega-1\right)+\omega} \cdot(-1) \\
=180^{0}
\end{gathered}
$$

which on simplification yields,

$$
\begin{gathered}
\frac{y}{x}=\frac{2 x y+y\left(\alpha_{t-1}-\omega-1\right)}{x^{2}-y^{2}+x\left(\alpha_{t-1}-\omega-1\right)+\omega} \\
\Rightarrow \mathrm{x}^{2}+\mathrm{y}^{2}=\omega
\end{gathered}
$$

This is an equation of circle with radius $\sqrt{\omega}$ and centre at $(0,0)$. The breakaway point is obtained by Setting

$$
\begin{gathered}
\frac{d \alpha^{l}{ }_{t-1}}{d z}=0, \text { where } \\
\alpha_{t-1}^{l}=\frac{z^{2}+z\left(\alpha_{t-1}-\omega-1\right)+\omega}{-z} \\
\frac{d \alpha^{l}{ }_{t-1}}{d z}=\frac{z\left(2 z+\alpha_{t-1}-\omega-1\right)-\left(z^{2}+z\left(\alpha_{t-1}-\omega-1\right)+\omega\right.}{z^{2}}=0 \\
\Rightarrow z=\sqrt{\omega}
\end{gathered}
$$

It is apparent from equation (26) that the root locus plots for the given transfer function is a circle with centre at origin and radius $=\sqrt{\omega}$. A series of root locus plots has been undertaken for different $\alpha_{t-1}$, the results of which for the two alternative closed loop systems Figures 2 (b) and (3) are presented in Tables 1 and 2 below. It is indicative from the four tables that the PSO dynamics is stable for $\alpha_{t-1}^{l}$ in [0,

0.103]. Consequently $\alpha_{t-1}^{g}$ appears to have a range:[0,(4 $0.103)]=[0,3.897]$. This is one of the primary achievements of this paper, which to the best of the authors' knowledge can uniquely determine the valuation space for the acceleration coefficients to ensure the stability of the PSO dynamics. The selection of $\alpha_{t-1}^{l}$ from the root locus plot was made based on the location of the dominant poles (complex conjugate poles close to the origin [6]). Naturally the selected values of $\alpha_{t-1}^{l}$ represents the DC gain of the closed loop system, when it is approximated by the pair of complex conjugate dominant poles. The root locus plots for stability analysis are shown in Figures 4(a), 4(b) and 4(c).

\section{VI.EXPERIMENTAL RESULTS}

In this Section, we present the results of computer simulation for the basic PSO algorithm outlined in section 2. omputer simulation of the basic PSO algorithm was undertaken on an IBM Pentium machine in C-language under Linux environment. A test suit containing 21 well known benchmark functions [12] were considered to study the performance analysis of the basic PSO algorithm with the selected parameter range as obtained from the root locus technique. Here, due to space limitations we are presenting the results only for Schaffer's $f_{6}$ function.

The Schaffer's $f_{6}$ is given by:

$$
f_{6}(x)=\sum_{i=1}^{D}\left(\left|x_{i}+0.5\right|\right)^{2}
$$

For performance evaluation, the average fitness of particles, and fitness of the best particle are used as the metrics, and the convergence in the two metrics over iterations is determined experimentally. The experiment was performed for different parameter settings of $\omega, \alpha_{t-1}^{l}$ and $\alpha_{t-1}^{g}$. Simulation results confirm that for the selected range of $\alpha_{t-1}^{l}$ and $\alpha_{t-1}^{g}$, PSO converges much faster with very good average fitness than the cases when the parameters go outside the range. It is also noted that for the selected range of $\alpha_{t-1}^{l}$ and $\alpha_{t-1}^{g}$, the average fitness (as well as the fitness of the best particle) increases at a faster rate than the cases where parameters are selected outside this range. The experimental results indicate that for all the typical 21 functions, $\omega=0.6, \alpha_{t-1}^{l}=0.103, \alpha_{t-1}^{g}=2.897$ yields the best results, i.e. the smallest convergence time with good fitness value. Figure 5 and Table 3 confirm the results. Consequently, the simulation reveals that the predicted acceleration coefficients and inertia factor play a significant role in controlling the stabilization behavior of the PSO dynamics. 

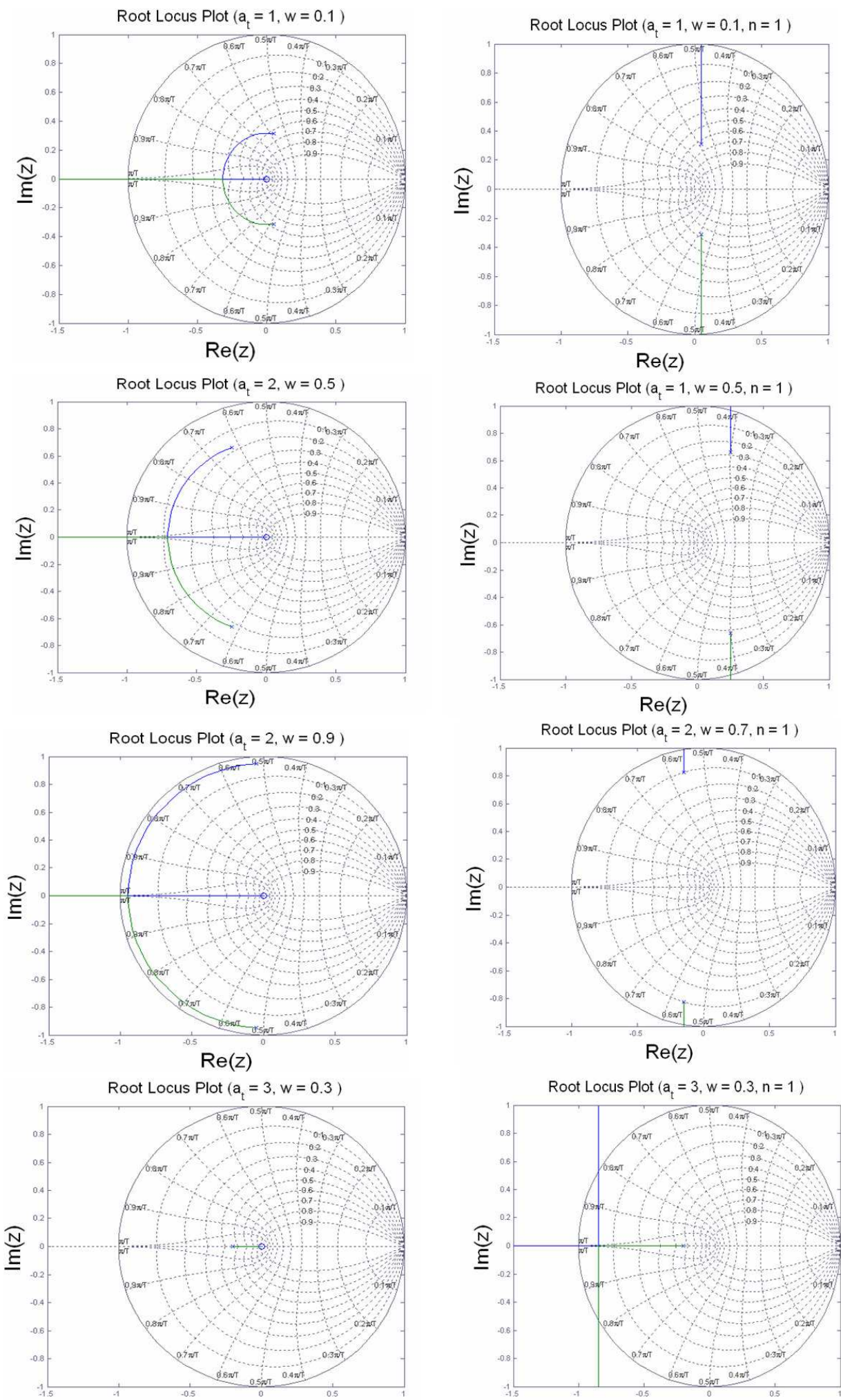

$\operatorname{Re}(z)$

Fig. 4(a) Root Locus analysis for stability when $H(z)=-1$

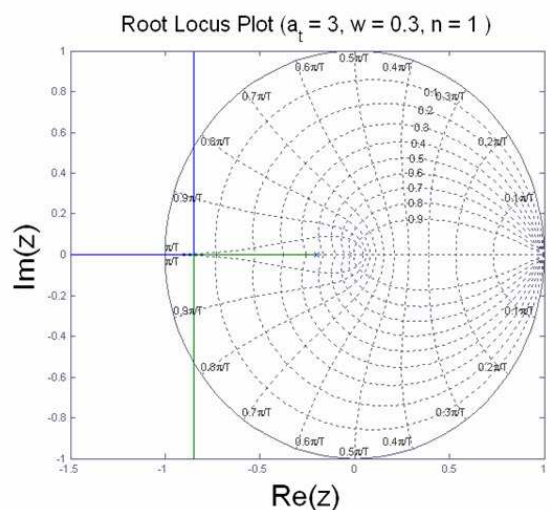

Fig. 4(b) Root Locus analysis for stability when $H(Z)=-Z^{-1}$ 

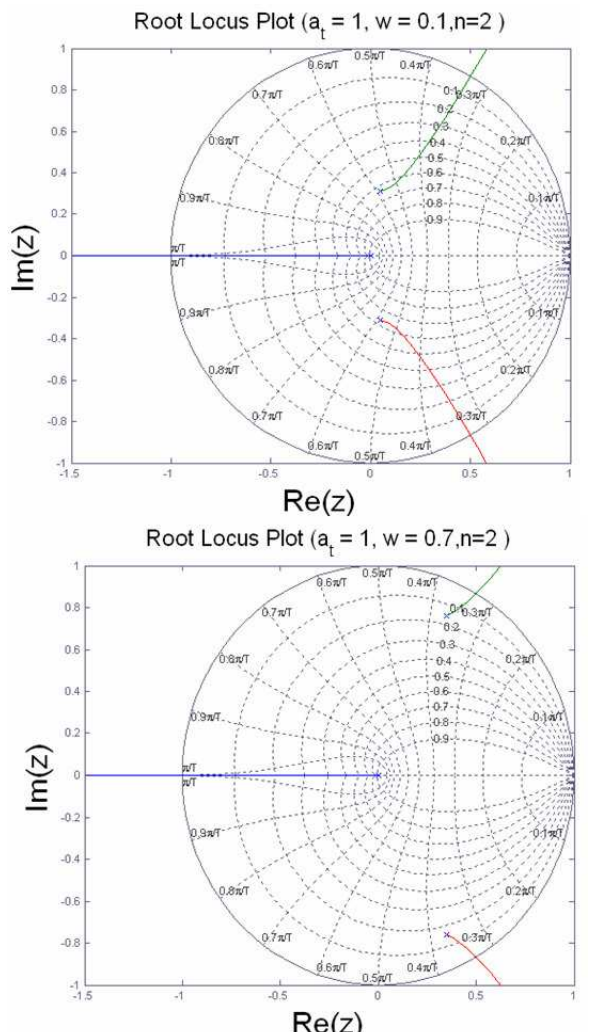

Root Locus Plot $\left(a_{t}=2, w=0.9, n=2\right)$

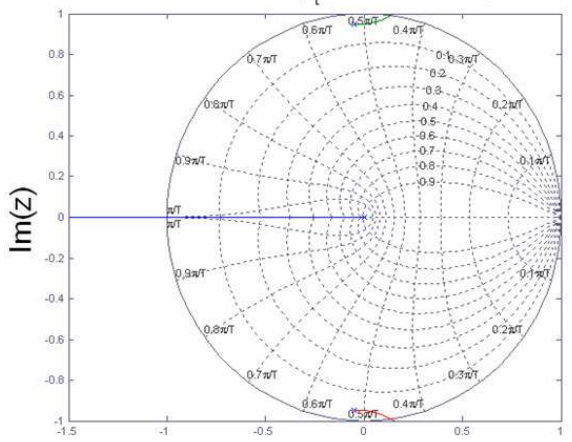

$\operatorname{Re}(z)$

Root Locus Plot $\left(a_{t}=3, w=0.3, n=2\right)$

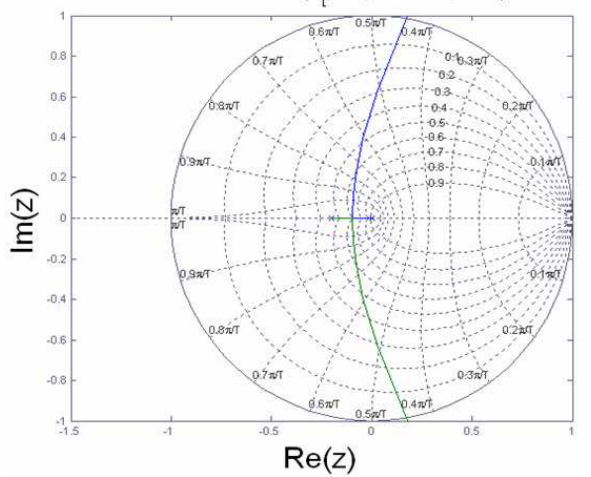

Fig. 4(c) Root Locus analysis for stability when $H(Z)=-Z^{-2}$
TABLE-1

Results of Stability Analysis with $\mathrm{H}(\mathrm{Z})=-1$

\begin{tabular}{|c|c|c|c|c|c|c|}
\hline \multirow[b]{2}{*}{$\alpha_{t-1}$} & \multirow[b]{2}{*}{$\omega$} & \multicolumn{2}{|c|}{ Dominant Roots } & \multirow[b]{2}{*}{$\left(\alpha_{t-1}^{l}\right)_{\mathrm{ma}}$} & \multirow[b]{2}{*}{$\alpha_{t-1}^{g}$} & \multirow[b]{2}{*}{ Stability } \\
\hline & & $\begin{array}{l}\lambda_{1}=-\delta+ \\
\sqrt{\delta^{2}-\omega}\end{array}$ & $\begin{array}{l}\lambda_{2}=-\delta- \\
\sqrt{\delta^{2}-\omega}\end{array}$ & & & \\
\hline 1 & 0.1 & $\begin{array}{c}0.05+ \\
\text { j0.312 }\end{array}$ & $0.05-\mathrm{j} 0.312$ & 1.0 & 0 & Stable \\
\hline 1 & 0.01 & $\begin{array}{l}0.025+ \\
\text { j0.096 }\end{array}$ & $0.025-\mathrm{j} 0.096$ & 0.8 & 0.2 & Stable \\
\hline 2 & 0.9 & $\begin{array}{l}-0.05+ \\
\text { j0.947 }\end{array}$ & $-0.05-\mathrm{j} 0.947$ & 1.8 & 0.2 & Stable \\
\hline 2 & 0.8 & $\begin{array}{c}-0.1+ \\
\text { j0.995 }\end{array}$ & $-0.1-$ j0.995 & 1.6 & 0.4 & Stable \\
\hline 2 & 0.7 & $\begin{array}{l}-0.15+ \\
\text { j0.823 }\end{array}$ & $-0.15-\mathrm{j} 0.823$ & 1.4 & 0.6 & Stable \\
\hline 2 & 0.6 & $\begin{array}{c}-0.2+ \\
\text { j0.748 }\end{array}$ & $-0.2-\mathrm{j} 0.748$ & 1.2 & 0.8 & Stable \\
\hline 2 & 0.5 & $\begin{array}{l}-0.25+ \\
j 0.661\end{array}$ & $-0.25-\mathrm{j} 0.661$ & 1.0 & 1.0 & Stable \\
\hline 2 & 0.4 & $\begin{array}{c}-0.3+ \\
\text { j0.557 }\end{array}$ & $-0.3-\mathrm{j} 0.557$ & 0.8 & 1.2 & Stable \\
\hline 2 & 0.3 & $\begin{array}{l}-0.35+ \\
\text { j0.421 }\end{array}$ & $-0.35-\mathrm{j} 0.421$ & 0.6 & 1.4 & Stable \\
\hline 2 & 0.2 & $-0.4+\mathrm{j} 0.2$ & $-0.4-\mathrm{j} 0.2$ & 0.4 & 1.6 & Stable \\
\hline 2 & 0.1 & -0.77 & -0.13 & 0.2 & 1.8 & Stable \\
\hline 3 & 0.9 & $\begin{array}{l}-0.55+ \\
\text { j0.773 }\end{array}$ & $-0.55-\mathrm{j} 0.773$ & 0.8 & 2.2 & Stable \\
\hline 3 & 0.8 & $\begin{array}{c}-0.6+ \\
\text { j0.663 }\end{array}$ & $-0.6-j 0.663$ & 0.6 & 2.4 & Stable \\
\hline 3 & 0.7 & $\begin{array}{l}-0.65+ \\
\text { j0.527 }\end{array}$ & $-0.65-\mathrm{j} 0.527$ & 0.4 & 2.6 & Stable \\
\hline 3 & 0.6 & $\begin{array}{c}-0.7+ \\
\text { j0.332 }\end{array}$ & $-0.7-\mathrm{j} 0.332$ & 0.2 & 2.8 & Stable \\
\hline 3 & 0.5 & -1.0 & -0.5 & 0 & 3.0 & Oscillatory \\
\hline
\end{tabular}

Where $\left(\alpha_{t-1}^{l}\right)_{\max }=\left|\frac{\sum_{j} z_{0}-p_{j}}{\sum_{i} z_{0}-p_{i}}\right|, \mathrm{z}_{0}=$ point on the unit circle, and $\alpha_{t-1}^{g}=\alpha_{t-1}-\left(\alpha_{t-1}^{l}\right)_{\max }$

TABLE 3

Parameter selection of PSO for the best fitness and the average fitness values obtained from Figure 5

\begin{tabular}{|l|c|c|c|c|c|c|}
\hline Roots & $\left(\alpha_{t-1}^{l}\right)_{\max }$ & $\alpha_{t-1}^{g}$ & $\boldsymbol{\omega}$ & Best fitnes & $\begin{array}{c}\text { Average } \\
\text { fitness }\end{array}$ & $\begin{array}{c}\text { System } \\
\text { stability }\end{array}$ \\
\hline $\begin{array}{l}-0.77, \\
-0.13, \\
0,0\end{array}$ & 0.478 & 1.522 & 0.1 & 0.32071 & 11.74614 & $\begin{array}{c}\text { Stable } \\
\text { overdamped }\end{array}$ \\
\hline $\begin{array}{l}-1.0, \\
-0.5,0,0\end{array}$ & 0.2 & 2.8 & 0.5 & - & - & $\begin{array}{l}\text { Sustained } \\
\text { oscillation }\end{array}$ \\
\hline $\begin{array}{l}-0.77, \\
-0.13, \\
0\end{array}$ & 0.321 & 1.679 & 0.1 & 0.036931 & 12.2793 & Stable \\
\hline $\begin{array}{l}-0.7 \pm \\
\text { j0.322, } \\
0\end{array}$ & $\mathbf{0 . 1 0 3}$ & $\mathbf{2 . 8 9 7}$ & $\mathbf{0 . 6}$ & 11.6891 & 28.7136 & Fast stable \\
\hline $\begin{array}{l}0.005 \pm \\
\text { j0.09, }\end{array}$ & 0.988 & 0.012 & 0.01 & 3.6363 & 16.7473 & stable \\
\hline
\end{tabular}


TABLE 2

Results of Stability Analysis with $H(Z)=-Z^{-1}$
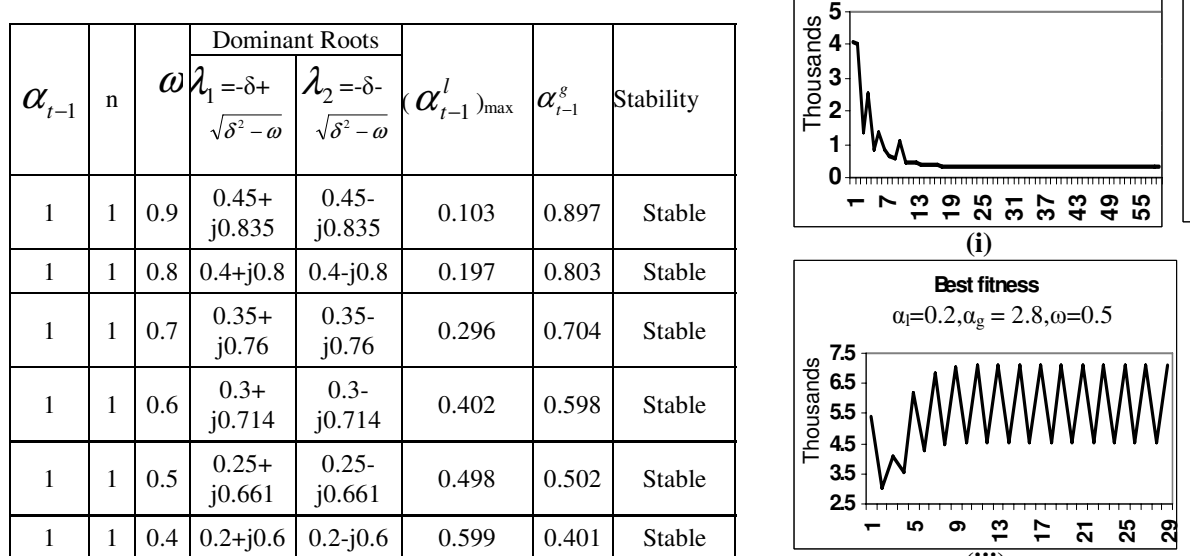

(iii)

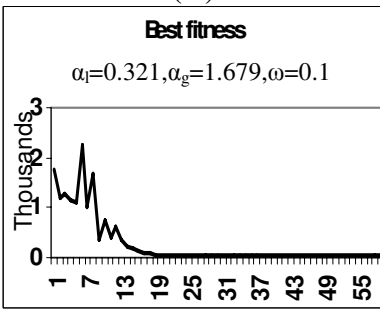

(v)

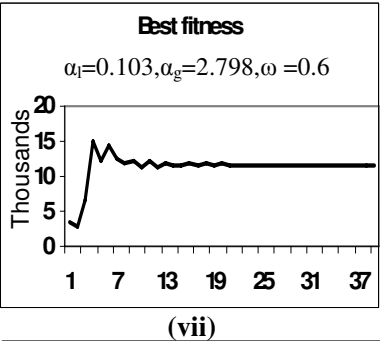

Best fitress

$\alpha_{\mathrm{l}}=0.988, \alpha_{\mathrm{g}}=0.012, \omega=0.01$

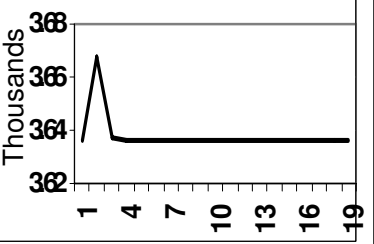

(ix)

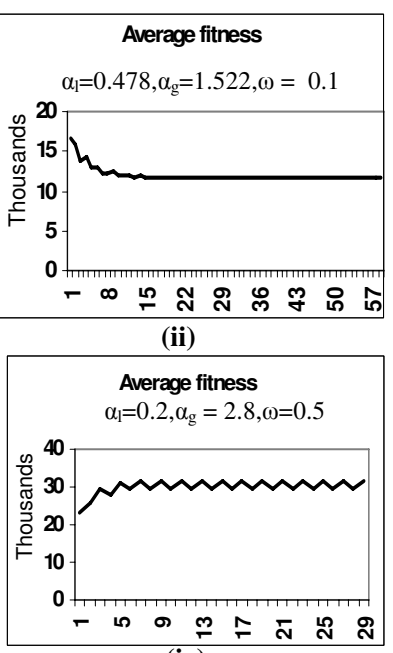

(iv)

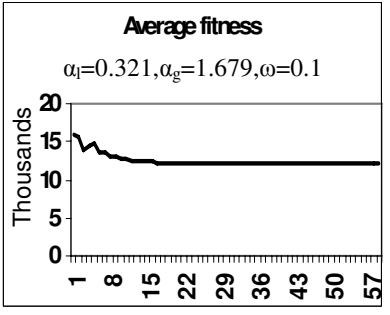

(vi)

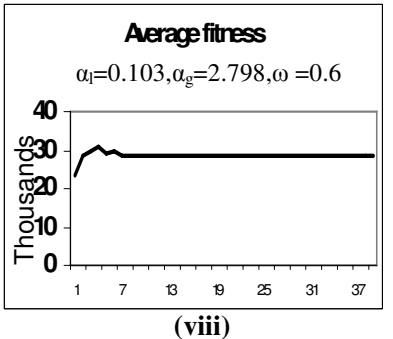

Averagefituess

$\alpha_{\mathrm{l}}=0.988, \alpha_{\mathrm{g}}=0.012, \omega=0.01$

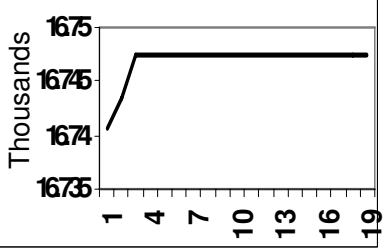

(x)

Fig.5: Results of execution of the PSO algorithm on Schaffer's $f_{6}$ for the parameter set mentioned in individual graphs. Best fitness and average fitness values $\left(\times 10^{3}\right)$ are plotted against iterations to demonstrate relative performance in convergence, supporting results of root locus analysis. 


\section{VII.CONCLUSIONS}

The paper proposed a novel approach to closed loop stability analysis of the PSO dynamics by Jury's test and root locus technique. Jury's stability test confirms established range for the inertia factor $(\omega)$, but also provides a new range for the acceleration co-efficient $\alpha_{t-1}^{g}$ in $[0,2(1+\omega)]$. The root locus analysis envisages that for two possible forms of non-linearity, the PSO dynamics is stable for $\alpha_{t-1}^{l}$ in [0, 0.103]. Computer simulation of the PSO algorithm confirmed the above results, and further indicate that $\omega=0.6, \alpha_{t-1}^{g}=2.897$ and $\alpha_{t-1}^{l}=0.103$ is the best choice for all test functions $f_{1}$ through $f_{21}$, and thus the PSO may alternatively be executed as a deterministic optimization algorithm with a fast convergence speed without any sacrifice in accuracy.

\section{REFERENCES}

[1] R. C. Eberhart and J. Kennedy, "A new optimizer using particle swarm theory," in Proceedings of $6^{\text {th }}$ Int. Symp. Micromachine Human Sci., vol. 1, 1995, pp. 39-43.

[2] Y. Shi and R. C. Eberhart, "Empirical study of particle swarm optimization," in Proceedings of IEEE Congress in Evolutionary Computation 1999, pp 1945-1950.

[3] M. Clerc and J. Kennedy, "The particle swarm-explosion, stability and convergence in a multidimensional complex space," IEEE Trans. Evol. Comput., vol.6, no.2, pp. 58-73, 2002.

[4] V.Kadirkamanathan, K.Selvarajah, P.J.Fleming, "Stability analysis of the particle dynamics in particle swarm optimizer," IEEE Trans. Evol. Comput., vol.10, no.3, pp. 245-255, 2006.

[5] B. C. Kuo, Digital Control System, Oxford, NY, 1992.

[6] M. Broae and N. Miphail, "Design of Crane Control Law," Control Engineering Courseware, Virtual Laboratory, 2002.

[7] F. Van den Bergh, "An analysis of particle swarm optimizers,"Ph.D. dissertation, Univ. Pretoria, South Africa, 2002.

[8] J. Kennedy, "The behavior of particle," in Proc. $7^{\text {th }}$ Annu. Conf. Evol. Program. San Diego, CA, 1998, pp. 581-589.

[9] R. C. Eberhart and Y. Shi, "Parameter selection in particle swarm optimization," in Proc. $7^{\text {th }}$ Conf. Evol. Program. vol. 1447, 1998, pp. 591-600.

[10] H. M. Emara and H. A. A. Fattah, "Continuous swarm optimization technique with stability analysis," in Proc. Amer. Conrol Conf. , Boston , MA, 2004, pp. 2811-2817.

[11] I. C. Trelea, "The particle swarm optimization algorithm: Convergence analysis and parameter selection," Inf. Process. Lett. Vol. 85, pp. 317-325, 2003.

[12] J. Brest, S. Greiner, B. Boskovic, M. Mernik and V. Zumer, "Selfadapting control parameters in differential evolution: a comparative study on numerical benchmark problems," IEEE Trans. Evol. Comput., vol.10, no.6, pp. 58-73, 2006. 